FACTA UNIVERSITATIS (NIŠ)

Ser. Math. Inform. Vol. 34, No 5 (2019), 837-847

https://doi.org/10.22190/FUMI1905837G

\title{
CURVES OF CONSTANT BREADTH ACCORDING TO DARBOUX FRAME IN GALILEAN SPACE $G_{3}$
}

\author{
Hülya Gün Bozok
}

(C) 2019 by University of Niš, Serbia | Creative Commons Licence: CC BY-NC-ND

\begin{abstract}
In this work, the curves of constant breadth according to Darboux frame in the 3-dimensional Galilean Space are investigated. Firstly the curves of constant breadth according to Darboux frame are determined then the differential equation of the constant breadth curve with this frame is found. After that some special cases of this differential equation are researched.
\end{abstract}

Keywords: Darboux frame; Galilean Space; constant breadth curve; differential equation.

\section{Introduction}

In 1780, Euler determined curves of constant breadth in the plane [13]. Constant breadth curves are an important issue not only in geometry but also in engineering sciences, especially, in cam designs [10]. Furthermore, the method related to the curves of constant breadth for the kinematics of machinery is given by Reuleaux [6]. Fujiwara introduced constant breadth for space curves and surfaces [14] and Struik made important publications on this subject [4]. After that in different spaces some important papers are published, such that in Euclidean 3-space Kose expressed some characterizations for space curves of constant breadth[16], Magden and Kose extended these curves in Euclidean 4-space [1]. Then using different frame these curves are researched in Euclidean space $[3,7,8]$. Characterizations for spacelike curves of constant breadth in Minkowski 4-space are given by Kazaz et al. [15]. The curve of constant breadth on the sphere studied by Blaschke [20]. The properties of these curves in 3-dimensional Galilean space and pseudo-Galilean space are given by Yoon and Unluturk et al., respectively [5, 21].

In the differential geometry especially theory of surfaces Darboux frame has an important role, a Darboux frame is a natural moving frame constructed on a

Received August 27, 2018; accepted October 30, 2018

2010 Mathematics Subject Classification. 53A35 
surface. It is the analog of the Frenet-Serret frame as applied to surface geometry. After determination of this frame a lot of work have been done in different spaces $[3,19]$.

In recent years, many researchers investigated the curves and surfaces in Galilean space and thereafter psedou-Galilean space. Roshel studied the theory of the curves in Galilean space [17]. After that a lot of works have been done by some authors in these space $[2,9,21]$. In Galilean space a Darboux frame is defined as follows:

Definition 1.1. Let $S$ be a surface in $G_{3}$ and $\alpha$ be a curve on $S$. If $T$ denotes the unit tangent vector to $\alpha, n$ denotes the unit normal vector of $S$ at the point $\alpha(x)$ of $\alpha$, and $Q=n \times_{G} T$ denotes the tangential normal. Then $\{T, Q\}$ is the basis for the vectors tangent to $S$ at $\alpha(x)$ and $\{T, Q, n\}$ is the orthonormal basis for all vectors at $\alpha(x)$ in $G_{3}$. Thus there is a new frame at every point of a curve on a surface $S$ in $G_{3}$ is constructed other than the Frenet-Serret frame. This frame is called Galilean Darboux frame or tangent-normal frame [18].

Theorem 1.1. Let $\alpha: I \subset \mathbb{R} \longrightarrow M \subset G_{3}$ be a unit speed curve and let $T, Q, n$ be the Darboux frame field of $\alpha$ with respect to $M$, then

$$
\left[\begin{array}{l}
T \\
Q \\
n
\end{array}\right]^{\prime}=\left[\begin{array}{ccc}
0 & \kappa_{g} & \kappa_{n} \\
0 & 0 & \tau_{g} \\
0 & -\tau_{g} & 0
\end{array}\right]\left[\begin{array}{l}
T \\
Q \\
n
\end{array}\right] .
$$

where $\kappa_{g}, \kappa_{n}$ and $\tau_{g}$ are geodesic curvature, normal curvature and geodesic torsion, respectively [18].

Also using the equation (1.1) the following relations are found,

$$
\begin{aligned}
\kappa^{2}(x) & =\kappa_{g}^{2}(x)+\kappa_{n}^{2}(x) \\
\tau(x) & =-\tau_{g}(x)+\frac{\kappa_{g}^{\prime}(x) \kappa_{n}(x)-\kappa_{g}(x) \kappa_{n}^{\prime}(x)}{\kappa_{g}^{2}(x)+\kappa_{n}^{2}(x)}
\end{aligned}
$$

where $\kappa(x)$ and $\tau(x)$ are the curvature and torsion of $\alpha$, respectively.

In this paper the curves of constant breadth according to Darboux frame in the 3-dimensional Galilean Space are researched. Then the curves of constant breadth according to Darboux frame are expressed and the differential equation of the constant breadth curve with this frame is obtained. After that some special cases of this differential equation are found.

\section{Preliminaries}

The Galilean space $G_{3}$ is a Cayley-Klein space equipped with the projective metric of signature $(0,0,+,+)$. The absolute figure of Galilean geometry consist of an ordered triple $\{w, f, I\}$, where $w$ is the ideal (absolute) plane, $f$ is the line (absolute line) in $w$ and $I$ is the fixed elliptic involution of points of $f$. 
Galilean scalar product can be written as

$$
\left\langle v_{1}, v_{2}\right\rangle= \begin{cases}x_{1} x_{2} & , \text { if } x_{1} \neq 0 \vee x_{2} \neq 0 \\ y_{1} y_{2}+z_{1} z_{2} & \text {, if } x_{1}=0 \wedge x_{2}=0\end{cases}
$$

where $v_{1}=\left(x_{1}, y_{1}, z_{1}\right)$ and $v_{2}=\left(x_{2}, y_{2}, z_{2}\right)$. It leaves invariant the Galilean norm of the vector $v=(x, y, z)$ defined by

$$
\|v\|= \begin{cases}x & , \text { if } x \neq 0 \\ \sqrt{y^{2}+z^{2}} & \text {, if } x=0\end{cases}
$$

[17]. If a curve $C$ of the class $C^{r}(r \geqslant 3)$ is given by the parametrization

$$
r=r(x, y(x), z(x))
$$

then $x$ is a Galilean invariant the $\operatorname{arc}$ length on $C$. The curvature and the torsion are expressed by,

$$
\kappa(x)=\sqrt{y^{\prime \prime 2}+z^{\prime \prime 2}}
$$

$$
\tau(x)=\frac{1}{\kappa^{2}(x)} \operatorname{det}\left(r^{\prime}(x), r^{\prime \prime}(x), r^{\prime \prime \prime}(x)\right) .
$$

The orthonormal trihedron is defined as follows:

$$
\begin{aligned}
t(x) & =\left(1, y^{\prime}(x), z^{\prime}(x)\right) \\
n(x) & =\frac{1}{\kappa(x)}\left(0, y^{\prime \prime}(x), z^{\prime \prime}(x)\right), \\
b(x) & =\frac{1}{\kappa(x)}\left(0,-z^{\prime \prime}(x), y^{\prime \prime}(x)\right) .
\end{aligned}
$$

The vectors $t, n, b$ are called the vectors of tangent, principal normal and binormal line of, respectively. For their derivatives the following Frenet formulas hold

$$
\begin{aligned}
t^{\prime}(x) & =\kappa(x) n(x) \\
n^{\prime}(x) & =\tau(x) b(x) \\
b^{\prime}(x) & =-\tau(x) n(x)
\end{aligned}
$$

[11]. For detailed information about the Galilean geometry we refer to $[12,17]$.

\section{Curves of Constant Breadth According Darboux Frame in $G_{3}$}

Let $X=\vec{X}(s)$ be a simple closed curve in 3-dimensional Galilean space $G_{3}$. These curves will be denoted by $(C)$. The normal plane at every point $P$ on the curve meets the curve at a single point $R$ other than $P$. The point $R$ is called the opposite point of $P$. Considering a curve $\alpha$ which have parallel tangents $\vec{T}$ and $\vec{T}^{*}$ in opposite points $X$ and $X^{*}$ of the curve as in [14]. A simple closed curve of constant breadth which have parallel tangents in opposite directions can be introduced by

$$
X^{*}\left(s^{*}\right)=X(s)+m_{1}(s) T(s)+m_{2}(s) Q(s)+m_{3}(s) n(s)
$$


where $T, Q, n$ denote the Darboux frame in 3-dimensional Galilean space $G_{3}$. Differentiating the equation (3.1) the following equation is obtained,

$$
\begin{aligned}
\frac{d X^{*}}{d s}=\frac{d X^{*}}{d s^{*}} \frac{d s^{*}}{d s}=T^{*} \frac{d s^{*}}{d s} & =\left(1+\frac{d m_{1}}{d s}\right) T \\
& +\left(\frac{d m_{2}}{d s}+m_{1} \kappa_{g}-m_{3} \tau_{g}\right) Q \\
& +\left(\frac{d m_{3}}{d s}+m_{1} \kappa_{n}+m_{2} \tau_{g}\right) n
\end{aligned}
$$

where $\kappa_{g}, \kappa_{n}$ and $\tau_{g}$ are geodesic curvature, normal curvature and geodesic torsion, respectively. Since $\left\langle T, T^{*}\right\rangle=-1$, the following relations are found,

$$
\begin{aligned}
\frac{d s^{*}}{d s}+\frac{d m_{1}}{d s}+1 & =0, \\
\frac{d m_{2}}{d s}+m_{1} \kappa_{g}-m_{3} \tau_{g} & =0, \\
\frac{d m_{3}}{d s}+m_{1} \kappa_{n}+m_{2} \tau_{g} & =0 .
\end{aligned}
$$

Assume that the angle between the tangents at the points $X(s)$ and $X(s+\Delta s)$ with $\Delta \phi$. If we denote the vector $T(s+\Delta s)-T(s)$ with $\Delta T$, then $\lim _{\Delta s \rightarrow 0} \frac{\Delta T}{\Delta s}=$ $\lim _{\Delta s \rightarrow 0} \frac{\Delta \phi}{\Delta s}=\frac{d \phi}{d s}=\kappa$. We called the angle of contingency to the angle $\Delta \phi$ [4]. So by using the equation $\frac{d \phi}{d s}=\kappa$, the equation (3.3) can be rewritten as follows:

$$
\begin{aligned}
\frac{d m_{1}}{d \phi} & =-f(\phi), \\
\frac{d m_{2}}{d \phi} & =\rho\left(m_{3} \tau_{g}-m_{1} \kappa_{g}\right), \\
\frac{d m_{3}}{d \phi} & =\rho\left(-m_{1} \kappa_{n}-m_{2} \tau_{g}\right),
\end{aligned}
$$

where $f(\phi)=\rho+\rho^{*}, \rho=\frac{1}{\kappa}$ and $\rho^{*}=\frac{1}{\kappa^{*}}$ denote the radius of curvatures at $X$ and $X^{*}$, respectively.

On the other hand, in differential geometry of surfaces for a curve $\alpha(s)$ lying on a surface there are following cases

i. $\alpha$ is a geodesic curve $\Longleftrightarrow \kappa_{g}=0$,

ii. $\alpha$ is a asymptotic line $\Longleftrightarrow \kappa_{n}=0$,

iii. $\alpha$ is a principal line $\Longleftrightarrow \tau_{g}=0$,

So, considering equation (3.4) some special cases of constant breadth curve with respect to Darboux frame are researched as follows:

\section{Case 1.}

Suppose that $X$ be a geodesic curve (not straight line) on surface then $\kappa_{g}=0$. 
Therefore using this statement and the equation (3.4) the following differential equation system can be written,

$$
\begin{aligned}
& m_{1}^{\prime}=-f(\phi), \\
& m_{2}^{\prime}=-\psi m_{3}, \\
& m_{3}^{\prime}=-m_{1}+\psi m_{2} .
\end{aligned}
$$

where $\psi=\frac{\tau}{\kappa}$. By using (3.5) the following differential equation is obtained,

$$
\begin{aligned}
m_{3}^{\prime \prime \prime} & -\frac{\psi^{\prime \prime}}{\psi^{\prime}} m_{3}^{\prime \prime}+\psi^{2} m_{3}^{\prime}+\left[-\frac{\psi^{\prime \prime}}{\psi^{\prime}} \psi^{2}+\psi^{\prime} \psi+\left(\psi^{2}\right)^{\prime}\right] m_{3} \\
& -f^{\prime}(\phi)+\frac{\psi^{\prime \prime}}{\psi^{\prime}} f(\phi)=0
\end{aligned}
$$

where $\psi=\frac{\tau}{\kappa}$. Assuming that $\left(X, X^{*}\right)$ pair of curve is constant breadth and using the equation $(2.2)$

$$
\left\|X^{*}-X\right\|^{2}= \begin{cases}m_{1}^{2}=\text { constant } & m_{1} \neq 0 \\ m_{2}^{2}+m_{3}^{2}=\text { constant } & m_{1}=0\end{cases}
$$

is obtained. Hence, the following relations are found,

$$
\begin{aligned}
m_{1} \frac{d m_{1}}{d \phi} & =0, \\
m_{2} \frac{d m_{2}}{d \phi}+m_{3} \frac{d m_{3}}{d \phi} & =0,
\end{aligned}
$$

where $m_{1} \neq 0$ and $m_{1}=0$, respectively. By considering system (3.5),(3.7) and (3.8) the following two equations are gotten,

$$
\begin{aligned}
m_{1} f(\phi) & =0, \\
m_{1} m_{3} & =0,
\end{aligned}
$$

where $m_{1} \neq 0$ and $m_{1}=0$, respectively. Then, these situations can be considered with some subcases.

Case 1.1. The case of $m_{1} f(\phi)=0$ and $m_{1}=$ constant $=l \neq 0$. In this case since $m_{1} \neq 0$, it is seen that $f(\phi)=0$. So assume that the curve $X$ is a general helix that is $\psi=k=$ constant and substituting the $f(\phi)=0$ in the equation (3.6)

$$
m_{3}^{\prime \prime \prime}+k^{2} m_{3}^{\prime}=0
$$

is found. By solving this equation, $m_{2}$ and $m_{3}$ are obtained as follows:

$$
m_{3}=c_{1} \cos k \phi+c_{2} \sin k \phi+c_{3}
$$


and

$$
m_{2}=-c_{1} \sin k \phi+c_{2} \cos k \phi+c_{3} \phi+c_{4}
$$

where $c_{1}, c_{2}, c_{3}, c_{4}$ are real constants.

Case 1.2. The case of $m_{1} m_{3}=0$ and $m_{1}=0$. In this case since $m_{1}=0$, it is seen that $m_{3}=0$ or $m_{3} \neq 0$. If $m_{1}=0$ and $m_{3}=0$ then $m_{2}=0$, so the curves $X$ and $X^{*}$ coincide. On the other hand if $m_{1}=0$ and $m_{3} \neq 0$ and if suppose that the curve $X$ is a general helix that is $\psi=k=$ constant and considering this situation in the equation (3.6)

$$
m_{3}^{\prime \prime \prime}+k^{2} m_{3}^{\prime}=0 .
$$

is obtained. By solving this equation

$$
m_{3}=c_{1} \cos k \phi+c_{2} \sin k \phi+c_{3}
$$

and

$$
m_{2}=-c_{1} \sin k \phi+c_{2} \cos k \phi+c_{3} \phi+c_{4}
$$

are found, where $c_{1}, c_{2}, c_{3}, c_{4}$ are real constants. Therefore the following theorem can be given:

Theorem 3.1. Suppose that $X(s)$ is a geodesic curve, is a general helix and $\left(X, X^{*}\right)$ be a pair of constant curve. Then the curve $X^{*}\left(s^{*}\right)$ can be expressed as follows

i. If $m_{1}=$ constant $=l \neq 0$

$$
X^{*}\left(s^{*}\right)=X(s)+m_{1} T(s)+m_{2} Q(s)+m_{3} n(s)
$$

where

$$
\begin{aligned}
& m_{1}=l=\text { constant }, \\
& m_{2}=-c_{1} \sin k \phi+c_{2} \cos k \phi+c_{3} \phi+c_{4}, \\
& m_{3}=c_{1} \cos k \phi+c_{2} \sin k \phi+c_{3} .
\end{aligned}
$$

ii. If $m_{1}=0$

$$
X^{*}\left(s^{*}\right)=X(s)+m_{1} T(s)+m_{2} Q(s)+m_{3} n(s)
$$

where

$$
\begin{aligned}
& m_{1}=0, \\
& m_{2}=-c_{1} \sin k \phi+c_{2} \cos k \phi+c_{3} \phi+c_{4}, \\
& m_{3}=c_{1} \cos k \phi+c_{2} \sin k \phi+c_{3} .
\end{aligned}
$$




\section{Case 2.}

Suppose that $X$ be a asymptotic line (not straight line) on surface then $\kappa_{n}=0$. Therefore using this statement and the equation (3.4) the following differential equation system can be written,

$$
\begin{aligned}
m_{1}^{\prime} & =-f(\phi), \\
m_{2}^{\prime} & =-\psi m_{3}-m_{1}, \\
m_{3}^{\prime} & =\psi m_{2} .
\end{aligned}
$$

where $\psi=\frac{\tau}{\kappa}$. By using (3.19) the following equation is obtained,

$$
\begin{aligned}
m_{2}^{\prime \prime \prime} & -\frac{\psi^{\prime \prime}}{\psi^{\prime}} m_{2}^{\prime \prime}+\psi^{2} m_{2}^{\prime}+\left[-\frac{\psi^{\prime \prime}}{\psi^{\prime}} \psi^{2}+\psi^{\prime} \psi+\left(\psi^{2}\right)^{\prime}\right] m_{2} \\
& -f^{\prime}(\phi)+\frac{\psi^{\prime \prime}}{\psi^{\prime}} f(\phi)=0
\end{aligned}
$$

Suppose that $\left(X, X^{*}\right)$ pair of curve is constant breadth, then using the equation (2.2) the following statement can be expressed,

$$
\left\|X^{*}-X\right\|^{2}= \begin{cases}m_{1}^{2}=\text { constant } & m_{1} \neq 0 \\ m_{2}^{2}+m_{3}^{2}=\text { constant } & m_{1}=0\end{cases}
$$

Hence,

$$
\begin{aligned}
m_{1} \frac{d m_{1}}{d \phi} & =0, \\
m_{2} \frac{d m_{2}}{d \phi}+m_{3} \frac{d m_{3}}{d \phi} & =0,
\end{aligned}
$$

are found, where $m_{1} \neq 0$ and $m_{1}=0$, respectively. By considering the system $(3.19),(3.21)$ and (3.22) the following two equations are obtained,

$$
\begin{aligned}
m_{1} f(\phi) & =0, \\
m_{1} m_{2} & =0,
\end{aligned}
$$

where $m_{1} \neq 0$ and $m_{1}=0$, respectively. Then, these situations can be considered with some subcases.

Case 2.1. The case of $m_{1} f(\phi)=0$ and $m_{1}=$ constant $=l \neq 0$. In this case since $m_{1} \neq 0$, it is seen that $f(\phi)=0$. So assume that the curve $X$ is a general helix that is $\psi=k=$ constant and substituting the $f(\phi)=0$ in the equation (3.20)

$$
m_{2}^{\prime \prime \prime}+k^{2} m_{2}^{\prime}=0
$$

is found. By solving the above equation

$$
m_{2}=c_{1} \cos k \phi+c_{2} \sin k \phi+c_{3}
$$


and

$$
m_{3}=c_{1} \sin k \phi-c_{2} \cos k \phi+c_{3} \phi+c_{4}
$$

are obtained, where $c_{1}, c_{2}, c_{3}, c_{4}$ are real constants.

Case 2.2. The case of $m_{1} m_{2}=0$ and $m_{1}=0$. In this case since $m_{1}=0$, it is seen that $m_{2}=0$ or $m_{2} \neq 0$. If $m_{1}=0$ and $m_{2}=0$ then $m_{3}=0$, so the curves $X$ and $X^{*}$ coincide. On the other hand if $m_{1}=0$ and $m_{2} \neq 0$ and if suppose that the curve $X$ is a general helix that is $\psi=k=$ constant and considering this situation in the equation (3.20) the following equation is obtained,

$$
m_{2}^{\prime \prime \prime}+k^{2} m_{3}^{\prime}=0 .
$$

By solving this equation

$$
m_{2}=c_{1} \cos k \phi+c_{2} \sin k \phi+c_{3}
$$

$$
m_{3}=c_{1} \sin k \phi-c_{2} \cos k \phi+c_{3} \phi+c_{4}
$$

are found, where $c_{1}, c_{2}, c_{3}, c_{4}$ are real constants. Therefore the following theorem can be given:

Theorem 3.2. Let $X$ be a asymptotic line, is a general helix and suppose that $\left(X, X^{*}\right)$ be a pair of constant curve. Then the curve $X^{*}\left(s^{*}\right)$ can be expressed as follows

i. If $m_{1}=$ constant $=l \neq 0$

$$
X^{*}\left(s^{*}\right)=X(s)+m_{1} T(s)+m_{2} Q(s)+m_{3} n(s)
$$

where

$$
\begin{aligned}
& m_{1}=l=\text { constant } \\
& m_{2}=c_{1} \cos k \phi+c_{2} \sin k \phi+c_{3}, \\
& m_{3}=c_{1} \sin k \phi-c_{2} \cos k \phi+c_{3} \phi+c_{4} .
\end{aligned}
$$

ii. If $m_{1}=0$

$$
X^{*}\left(s^{*}\right)=X(s)+m_{1} T(s)+m_{2} Q(s)+m_{3} n(s)
$$

where

$$
\begin{aligned}
& m_{1}=0 \\
& m_{2}=c_{1} \cos k \phi+c_{2} \sin k \phi+c_{3}, \\
& m_{3}=c_{1} \sin k \phi-c_{2} \cos k \phi+c_{3} \phi+c_{4} .
\end{aligned}
$$




\section{Case 3.}

Suppose that $X$ be a principal line (not straight line) on surface then $\tau_{g}=0$. Therefore using this statement and the equation (3.4) the following differential equation system can be written,

$$
\begin{aligned}
& m_{1}^{\prime}=-f(\phi), \\
& m_{2}^{\prime}=-m_{1} \cos \phi, \\
& m_{3}^{\prime}=-m_{1} \sin \phi .
\end{aligned}
$$

Suppose that $\left(X, X^{*}\right)$ pair of curve is constant breadth, then using the equation

$$
\left\|X^{*}-X\right\|^{2}= \begin{cases}m_{1}^{2}=\text { constant } & m_{1} \neq 0 \\ m_{2}^{2}+m_{3}^{2}=\text { constant } & m_{1}=0\end{cases}
$$

is obtained. Thereafter the following two equations can be expressed

$$
\begin{aligned}
m_{1} \frac{d m_{1}}{d \phi} & =0 \\
m_{2} \frac{d m_{2}}{d \phi}+m_{3} \frac{d m_{3}}{d \phi} & =0
\end{aligned}
$$

where $m_{1} \neq 0$ and $m_{1}=0$, respectively. By considering system (3.33),(3.34) and (3.35) the following two equations are found,

$$
\begin{aligned}
m_{1} f(\phi) & =0, \\
m_{1} m_{2} & =0,
\end{aligned}
$$

where $m_{1} \neq 0$ and $m_{1}=0$, respectively. So, these situations can be considered with some subcases.

Case 3.1. The case of $m_{1} f(\phi)=0$ and $m_{1}=$ constant $=l \neq 0$. In this case since $m_{1} \neq 0$, it is seen that $f(\phi)=0$. So,

$$
m_{2}=-l \sin \phi+c_{1}
$$

$$
m_{3}=l \cos \phi+c_{2}
$$

are found, where $c_{1}, c_{2}$ are real constants.

Case 3.2. The case of $m_{1}=0$. In this case since $m_{1}=0$, it is seen that $m_{2}^{\prime}=0$ and $m_{3}^{\prime}=0$. So $m_{2}=a=$ constant and $m_{3}=b=$ constant are obtained. 
Theorem 3.3. Let $X$ is a principal line and $\left(X, X^{*}\right)$ be a pair of constant curve. Then the curve $X^{*}\left(s^{*}\right)$ can be expressed as follows:

i. If $m_{1}=0$,

$$
X^{*}\left(s^{*}\right)=X(s)+a(s) Q(s)+b(s) n(s)
$$

where $a, b$ are real constants.

ii. If $m_{1} \neq 0$,

$$
X^{*}\left(s^{*}\right)=X(s)+l T+\left(-l \sin \phi+c_{1}\right) Q(s)+\left(l \cos \phi+c_{2}\right) n(s)
$$

where $l$ is real constants.

\section{REF E R E N C E S}

1. A. Magden and O.Kose: On the curves of constant breadth in $E^{4}$ space, Turkish J. Math., 21, (1997), 277-284.

2. A.O. Ogrenmis, M. Ergut, and M. Bektas: On the helices in the Galilean Space $G_{3}$, Iranian J. Sci. Tech. A., 31, (2007), 177-181.

3. B. Altunkaya and F. Kahraman Aksoyak: Curves of constant breadth according to Darboux frame, Commun. Fac. Sci. Univ. Ank. Series A1.,66, (2017), 44-52.

4. D.J. Struik: Differential geometry in the large, Bull. Amer. Math. Soc., 37, (1931), 49-62.

5. D.W. Yoon: Curves of constant breadth in Galilean 3-space, Applied Mathematical Sciences, 8, (2014), 7013-7018.

6. F. Reuleaux: The Kinematics of Machinery, Trans. By A. B. W. Kennedy, Dover, Pub. Nex York, (1963).

7. H. Gun BozoK and H. OzTEKIN: Some characterization of curves of constant breadth according to Bishop frame in $E^{3}$ space, i-manager's Journal on Mathematics, 2, (2013), $7-11$.

8. H. Gun Bozok, S. Aykurt Sepet and M. Ergut: Curves of constant breadth according to type-2 bishop frame in $E^{3}$, Commun. Fac. Sci. Univ. Ank. Ser. A1 Math. Stat., 66, (2017), 206-212.

9. H. Oztekin, T. Korpinar and G. Altay: Constant ratio curves in the four dimensional Galilean Space, South Asian Journal of Mathematics, 2, (2017), 148-154.

10. H. TANAKA: Kinematics Design of Cam Follower Systems, Ph. D. Thesis, University of Columbia , (1976).

11. I. Kamenarovic: Existence Theorems for Ruled Surfaces in the Galilean Space $G_{3}$. Rad HAZU Math., 10, (1991), 183-196.

12. I.M. Yaglom: A Simple Non-Euclidean Geometry and Its Physical Basis, SpringerVerlag, New York, (1979).

13. L. Euler: De curvis triangularibus, Acta Acad. Prtropol., (1780), 3-30.

14. M. FujivarA: On Space Curves of Constant Breadth, Tohoku Math. J., (1914), 179-184.

15. M. Kazaz, M. Onder and H. Kocayigit: Spacelike curves of constant breadth in Minkowski 4-space, Int. J. Math. Anal., 2,(2008), 1061-1068. 
16. O. Kose: On space curves of constant breadth, Doga Mat., 10, (1986), 11-14.

17. O.Röschel: Die Geometrie des Galileischen Raumes, Habilitationsschrift, Leoben, (1984).

18. T.SAHIN: Intrinsic equations for a generalized relaxed elastic line on an oriented surface in the Galilean space, Acta Mathematica Scientia, 33, (2013), 701-711.

19. U. Ozturk, E.B. Koc Ozturk, and E.Nesovic: On equiform Darboux helices in Galilean 3-space, Math. Commun., 23, (2018), 145-159.

20. W.BlaschKe: Konvexe Bereiche gegebener konstanter Breite und kleinsten Inhalts, Math. Ann., 76, (1915), 504-513.

21. Y. Unluturk, M. Dede, Ü.Z. SavcÝ and C. Ekici: On curves of constant breadth in $G_{3}^{1}$, TWMS J. App. Eng. Math., 6, (2016), 64-69.

\section{Hülya Gün Bozok}

Osmaniye Korkut Ata University

Faculty of Arts and Science

Department of Mathematics

80000 Osmaniye, Turkey

hulyagun@osmaniye.edu.tr 